\title{
TOXICIDADE AGUDA E HISTOPATOLOGIA DO FíGADO DE LARVAS DE TRAIRÃO (Hoplias lacerdae) EXPOSTAS À SOLUÇÃO AQUOSA DE FORMALDEÍDO A 10\%
}

\author{
CLAUDINEI DA CRUZ * \\ RODRIGO YUDI FUJIMOTO * \\ RONALD KENNEDY LUZ** \\ MARIA CÉLIA PORTELLA*** \\ MAURÍCIO LATERÇA MARTINS ****
}

O objetivo deste trabalho foi estimar a concentração letal $50 \%$ (CL (I) ${ }_{50-96 h}$ ) da solução aquosa de formaldeído a $10 \%$ para larvas de trairão (Hoplias lacerdae) e avaliar os aspectos histopatológicos no fígado das larvas expostas a concentrações subagudas. Para tanto, realizou-se experimento com seis concentrações crescentes $(0,0 ; 0,5 ; 1,0 ; 1,5$; 2,0 e 2,5 mL) de formaldeído. Após 96 horas de exposição foram coletadas as larvas sobreviventes de cada tratamento. As larvas foram fixadas em solução de bouin e incluídas em parafina plástica para a obtenção dos cortes histológicos de $5 \mu \mathrm{m}$ e coloração em hematoxilina/eosina. A CL (I) ${ }_{50-96 h}$ para larvas de trairão foi de $2,021 \mathrm{~mL} / \mathrm{L}$ e a mortalidade de $5 \%, 10 \%, 10 \%$ e $20 \%$ para os tratamentos com $0,5,1,0,1,5$ e 2,0 mL/L, respectivamente, após 96 horas de exposição e de $100 \%$ para o tratamento com 2,5 mL/L após $24 \mathrm{~h}$. Nos tratamentos com 1,0; 1,5 e $2,0 \mathrm{~mL} / \mathrm{L}$ verificou-se desarranjo cordonal dos hepatócitos, fusão celular e congestão nos sinusóides. A solução aquosa de formaldeído a $10 \%$ pode ser utilizada para terapêutica ou profilaxia em larvas de trairão, em concentrações de 0,$5 ; 1,0$ e $1,5 \mathrm{~mL} / \mathrm{L}$, devido a baixa mortalidade dos peixes, porém é necessário alguns cuidados com sua utilização devido sua moderada toxicidade aguda.

PALAVRAS-CHAVES: Hoplias lacerdae; TELEÓSTEO; TOXICIDADE AGUDA; FÍGADO; FORMALDEÍDO.

* Doutor em Aqüicultura de Águas Continentais, Centro de Aqüicultura da Universidade Estadual Paulista (CAUNESP) (e-mail: cruzcl@yahoo.com).

** Doutor em Aqüicultura de Águas Continentais, CAUNESP/UNESP, Campus de Jaboticabal. PósDoutorado na Universidad Complutense de Madrid, Facultad de Biologia, Espanha.

*** Doutora em Ciências, Ecologia e Recursos Naturais, Professora do Departamento de Biologia Aplicada da Faculdade de Ciências Agrárias e Veterinárias, UNESP, Campus de Jaboticabal.

**** Doutor em Aqüicultura de Águas Continentais, CAUNESP, Professor do Departamento de Aqüicultura da Universidade Federal de Santa Catarina (UFSC), Florianópolis. 


\section{INTRODUÇÃO}

A aqüicultura brasileira está passando por processo de intensificação da produção, cujo sucesso depende da tecnificação e do manejo intenso (FUJIMOTO e CARNEIRO, 2001). A adoção de manejos intensivos tem provocado modificações nos ecossistemas em que as pisciculturas estão inseridas, pois a elevada concentração de animais favorece $\mathrm{o}$ aparecimento de doenças. $O$ estresse em peixes pode ser favorecido pela alta densidade de estocagem, manejo e desinfecção, tratamentos, transporte, reprodução artificial e baixa qualidade da água (TORANZO et al. 2004).

A importância econômica dos parasitos na piscicultura é verificada de modo indireto mediante redução das taxas de assimilação e crescimento, diminuição do valor do produto final para a comercialização e mortalidade dos animais (EIRAS, 1994).

Tratamentos terapêuticos com produtos químicos para o controle de parasitos em animais de cultivos resultam na liberação de grandes quantidades de substâncias ativas e de seus metabólitos no ambiente aquático (KINKELIN, MICHEL e GHITTINO, 1992). Um dos produtos químicos mais utilizados na aqüicultura é o formaldeído. A solução aquosa de formaldeído é amplamente empregada como antifúngico e anti-parasitário (RACH et al. 1997), principalmente de protozoários e de monogenéticos. Sua utilização foi aprovada nos Estados Unidos pelo Food and Drugs Administration (FDA) para ser utilizada no controle de fungos, protozoários e parasitos externos (FDA, 1992 e 1998).

Alguns trabalhos sobre a eficácia do formaldeído têm sido realizados no Brasil, porém pouco se sabe sobre as concentrações ideais e os níveis de tolerância para as diversas espécies nativas brasileiras, além dos possíveis resíduos que podem ser lançados no ambiente aquático. Entre as doenças que são provocadas por parasitos, Ichthyophthirius multifiliis e helmintos monogenéticos são comuns e importantes por causarem grandes perdas no estabelecimento de pisciculturas de alevinagem e engorda. Essas doenças geralmente são tratadas com solução aquosa de formaldeído (JUNG et al. 2001).

Segundo MARTINS (2004), o formaldeído a 37\% pode ser utilizado na forma de banho de curta duração (até 60 minutos) na concentração de 150 a $250 \mathrm{~mL} / \mathrm{m}^{3}$ e em banhos de longa duração $(24 \mathrm{~h}$ ) na concentração de 10 a $15 \mathrm{~mL} / \mathrm{m}^{3}$. No entanto, ressalta que o formaldeído agride o epitélio das brânquias e diminui a concentração de oxigênio dissolvido na água. NOGA (1996) também observou que o formol agride as brânquias e que sua toxicidade aumenta em águas ácidas e com alta temperatura.

Os testes de toxicidade aguda são utilizados para detectar e avaliar a capacidade do agente tóxico em produzir efeitos deletérios nos organismos vivos (CETESB, 1999). Estudos sobre a toxicidade do formaldeído foram realizados por CRUZ e PITOGO (1989) em Chanos chanos, por REARDON e HARRELL (1990) em Morone saxatilis e por FAJER-ÁVILA et al. (2003) em Sphoeroides annulatus. Testes de toxicidade aguda também foram realizados com outros ingredientes ativos utilizados na piscicultura para o controle de parasitos, tais como o verde malaquita (SRIVASTAVA et al. 1995), o sulfato de cobre (REARDON e HARREL, 1990; HENARES, MACHADO-NETO e CRUZ, 2003), o permanganato de potássio (STRAUS, 2004) e a cloramina - T (ALTINOK, 2004).

Dentre as espécies de interesse econômico destaca-se o trairão (Hoplias lacerdae). As espécies da família Erythrinidae são animais de água doce que se distribuem nas regiões tropicais e subtropicais da América do Sul (PAIVA, 1974). O trairão é muito apreciado por pescadores e populações ribeirinhas por apresentar características para a pesca esportiva e pela qualidade de sua carne. Durante o processo de larvicultura, um dos problemas mais comuns em termos de sanidade é o surgimento de parasitoses. Assim, é preciso conhecer os medicamentos a serem utilizados para não prejudicar o desenvolvimento do animal e afetar o meio ambiente. Deste modo, os objetivos deste trabalho foram estimar a concentração letal (CL $\left.(\mathrm{I})_{50-96 h}\right)$ de formaldeído para larvas de trairão e avaliar os efeitos histopatológicos no fígado das larvas expostas a concentrações subagudas de formaldeído. 


\section{MATERIAL E MÉTODOS}

O experimento foi realizado no Laboratório de Patologia de Organismos Aquáticos do Centro de Aqüicultura da Universidade Estadual Paulista. Utilizou-se delineamento inteiramente casualizado com 6 tratamentos e 4 repetições. Os tratamentos foram compostos pelas concentrações de 0,$0 ; 0,5$; 1,$0 ; 1,5 ; 2,0$ e 2,5 mL/L de solução aquosa de formaldeído a 10\%. Assim, a concentração de ingrediente ativo equivaleu a 0,$0 ; 1,85 ; 3,70 ; 5,55 ; 7,40 ; 9,25 \mathrm{mg}$ de formaldeído/ $\mathrm{L}$ de água. Em cada parcela foram colocadas 20 larvas de trairão (com 12 dias de vida e quatro dias de alimentação exógena) com peso médio de 13,4 \pm 0,13 mg e comprimento total médio de 1,16 $\pm 0,09 \mathrm{~cm}$. O período inicial de 12 dias foi utilizado para a aclimatação dos animais conforme recomendado pelo IBAMA (1987) e pela APHA (1991).

O teste de toxicidade aguda foi realizado durante 96 horas, observando-se a mortalidade a cada seis horas e retirando-se as larvas mortas. Efetuou-se o experimento em sistema estático, ou seja, sem troca de água e sem aeração, com temperatura constante. Durante o experimento foram monitorados a temperatura da água, o oxigênio dissolvido e o $\mathrm{pH}$, os quais não foram limitantes para o crescimento e sobrevivência das larvas (Tabela 1). Os valores de CL (I) ${ }_{50-96 h}$ foram calculados pelo método Trimmed Spearman-Karber (HAMILTON et al. 1977). Após 96 horas de exposição foram coletadas as larvas sobreviventes de cada tratamento, as quais foram fixadas em bouin por 24 horas. A seguir, as larvas foram lavadas, conservadas em álcool 70\% e incluídas em parafina plástica (Histosec $^{\circledast}$, Merck). Após a inclusão, realizou-se a microtomia em micrótomo automático (Leica ${ }^{\circledR}$ RM-2155), obtendo-se cortes histológicos de $5 \mu \mathrm{m}$. Os cortes histológicos foram corados com hematoxilina/eosina de acordo com BEHMER, TOLOSA e FREITAS NETO (1976).

\section{RESULTADOS E DISCUSSÃO}

Os testes de toxicidade são importantes antes de se recomendar qualquer tipo de tratamento, como quimioterápico, porque alguns produtos apresentam concentração terapêutica próxima da letal e a tolerância a alguns produtos depende da condição em que se procede o tratamento (FAJER-AVILA et al. 2003). Assim, calculou-se a relação concentração-resposta da formaldeído para as larvas de Hoplias lacerdae pela equação linear $y=42,571 x-25,429\left(R^{2}=0,77\right)$. A concentração letal $50 \%(C L$ (I) ${ }_{50-96 \mathrm{~h}}$ ) estimada foi de $2,02 \mathrm{~mL}$ de solução aquosa de formaldeído a $10 \% / \mathrm{L}$ de água, equivalente a $7,47 \mathrm{mg}$ de formaldeído/L.

O formaldeído é pouco tóxico para os peixes de água doce segundo FAJER-AVILA et al. (2003), porém apresenta variação muito grande de $C L(\mathrm{I})_{50-96 \mathrm{~h}}$ de 26 a $1020 \mathrm{mg}$ de formaldeído a 37\%/L de água. A CL (I) ${ }_{50-96 h}$ estimada para o trairão enquadrou-se abaixo dessa faixa de variação, demonstrando que a larva é mais sensível. Para lagosta americana (Homarus americanus), as larvas foram mais tolerantes ao azametifós do que os adultos (BURRIDGE et al. 1999) e os embriões de Bufo arenarum expostos ao paration (ANGUIANO et al. 1994)

A solução aquosa de formaldeído a $10 \%$ foi menos tóxica que o verde malaquita para o Heteropneustes fossilis com $\mathrm{CL}_{50-96 \mathrm{~h}}$ de $1,0 \mathrm{mg} / \mathrm{L}$ (SRIVASTAVA et al. 1995; SRIVASTAVA, SINHA e ROY, 2004), que o paration para Danio rerio com 1,94 mg/L (ROEX, VAN LANGEN e VAN GESTEL, 2002), que a permetrina para adultos de Poecilia reticulata com $\mathrm{CL}_{50-48 \mathrm{~h}}$ de $245,7 \mu \mathrm{g} / \mathrm{L}$ (BASER et al. 2003) e que o sulfato de cobre para o Xiphophorus maculatus com 1,72 mg/L (HENARES, MACHADONETO e CRUZ, 2003).

O efeito tóxico da solução aquosa de formaldeído foi maior que o formaldeído para Oreochromis niloticus com $\mathrm{CL}_{50-96 h}$ de 429,6 mg/L (MAcNIVEN e LITTLE, 2001), que o permanganato de potássio com 2,97 mg/L no híbrido de Morone saxatilis (STRAUS, 2004) e que o paration metílico em Piaractus mesopotamicus com 3,97 mg/L para alevinos e 9,89 mg/L para jovens (CRUZ, MACHADO-NETO e 
MENEZES, 2004) e que a cloramina T com $24,3 \mathrm{mg} / \mathrm{L}$ (ALTINOK, 2004).

As variáveis de qualidade de água (oxigênio dissolvido, $\mathrm{pH}$ e temperatura de água) não foram limitantes para o crescimento e sobrevivência das larvas de trairão (Tabela 1). Após 24 horas de exposição verificou-se $100 \%$ de mortalidade das larvas na concentração de $2,5 \mathrm{~mL} / \mathrm{L}$. Nessa concentração, no período entre 0 a 24 h de exposição, as larvas apresentaram-se imóveis, sem reação a estímulos externos e com a coloração esbranquiçada. Também apresentaram pequeno filme opaco em volta do corpo e erosão das nadadeiras peitorais. A presença de erosão nas nadadeiras também foi relatada por FAJER-ÁVILA et al. (2003) para o Sphoeroides annulatus, após 20 minutos de exposição a $142 \mathrm{mg} / \mathrm{L}$ de formaldeído. Esses sinais de intoxicação diferem dos observados por MURTY et al. (1984) para Mystus cavasius e por CRUZ, MACHADO-NETO e MENEZES (2004) para o P. mesopotamicus, expostos ao paration metílico. Segundo esses autores, durante a primeira hora de experimentação os peixes nadaram com a cabeça para cima ou para baixo continuamente, com sinais claros de intoxicação devido à ação do agrotóxico na inibição do sistema enzimático colinérgico. Para ALTINOK (2004) o Carassius auratus exposto à concentração de 20 mg/L de cloramina -T apresentou produção excessiva de muco, hiperventilação branquial e natação turbulenta na superfície da água. A maior incidência de morte dos animais expostos à solução aquosa de formaldeído ocorreu nas primeiras 24 horas de exposição, conforme já observado para o pacu ( $P$. mesopotamicus) exposto ao paration metílico e azadiractina (CRUZ, MACHADO-NETO e MENEZES, 2004).

\section{TABELA 1 - MORTALIDADE (\%) DE LARVAS DE TRAIRÃO (H. Lacerdae) E VARÁVEIS DE QUALIDADE DA ÁGUA DURANTE A DETERMINAÇÃO DA CONCENTRAÇÃO LETAL (CL (I) $\left.{ }_{50-96 H}\right)$ DO FORMALDEÍDO}

\begin{tabular}{c|c|c|c|c}
\hline $\begin{array}{c}\text { Concentração } \\
(\mathrm{mL} / \mathrm{L})\end{array}$ & $\begin{array}{c}\text { Mortalidade }(\%) \\
\text { após 96h }\end{array}$ & $\begin{array}{c}\text { Oxigênio dissolvido } \\
(\mathrm{mg} / \mathrm{L})\end{array}$ & $\mathrm{pH}$ & $\begin{array}{c}\text { Temperatura } \\
\text { da água }\left({ }^{\circ} \mathrm{C}\right)\end{array}$ \\
\hline 0,0 & 0 & 6,8 & 7,5 & $\mathbf{2 8 , 0 1}$ \\
\hline 0,5 & 5 & 6,5 & 7,6 & $\mathbf{2 8 , 0 3}$ \\
\hline 1,0 & 10 & 6,4 & 7,4 & $\mathbf{2 8 , 0 6}$ \\
\hline 1,5 & 10 & 6,0 & 7,5 & $\mathbf{2 8 , 0 4}$ \\
\hline 2,0 & 20 & 6,2 & 7,4 & $\mathbf{2 8 , 0 1}$ \\
\hline 2,5 & 100 & 6,1 & 7,6 & $\mathbf{2 8 , 0 3}$ \\
\hline
\end{tabular}

Segundo SHAO-NAN e DEFANG (1996), o malation foi considerado moderadamente tóxico para peixes com CL ${ }_{50-96 h}$ entre 0,25 e $15 \mathrm{mg} / \mathrm{L}$. Essa faixa de toxicidade também foi observada para as larvas de trairão expostas ao formaldeído a 10\%, porém sua concentração letal mostrou-se abaixo da constatada por FAJER-AVILA et al. (2003) que considerou o formaldeído pouco tóxico para peixes de água doce. Assim, são necessários cuidados na utilização do formaldeído em tratamentos terapêuticos e profiláticos de parasitos de trairão, devendo sempre ser realizado teste prévio nos animais. $O$ formaldeído foi considerado moderadamente tóxico para larvas de trairão (H. lacerdae), baseado na classificação de ZUCKER (1985), com a CL ${ }_{50}$ para peixes e invertebrados aquáticos variando entre 1 a $10 \mathrm{mg} / \mathrm{L}$.

\subsection{HISTOPATOLOGIA DO FÍGADO}

O fígado das larvas de trairão nos tratamentos controle e $0,5 \mathrm{~mL} / \mathrm{L}$ evidenciou organização cordonal do arranjo dos hepatócitos, formando placa de células em contato direto com os sinusóides. Os hepatócitos apresentaram forma hexagonal, com núcleo central, basófilo, cromatina descondensada e nucléolo visível. O citoplasma róseo indicou alta acidofilia e presença de alguns grânulos de glicogênio. Os sinusóides apresentaram-se revestidos por endotélio delgado com células endoteliais pequenas e 
núcleos proeminentes, além da presença de células sanguíneas dentro dos sinusóides (Figura 1). Segundo ROBERTS (1981), os peixes diferem dos mamíferos por não apresentarem sempre os hepatócitos em arranjo cordonal ou em lóbulos dos hepatócitos. No entanto, neste trabalho foi possível constatar o arranjo cordonal em larvas de trairão. Características histológicas semelhantes também foram descritas por SOUZA et al. (2001) em pacu (P. mesopotamicus).

FIGURA 1 - FOTOMICROGRAFIA DO FÍGADO DE TRAIRÃO (Hoplias lacerdae) EXPOSTO A 0,5 mL/L DE FORMALDEÍDO EVIDENCIANDO O ARRANJO CORDONAL DOS HEPATÓCITOS (TRAÇO) AO LONGO DO CAPILAR SINUSÓIDE (S) - H/E 400X

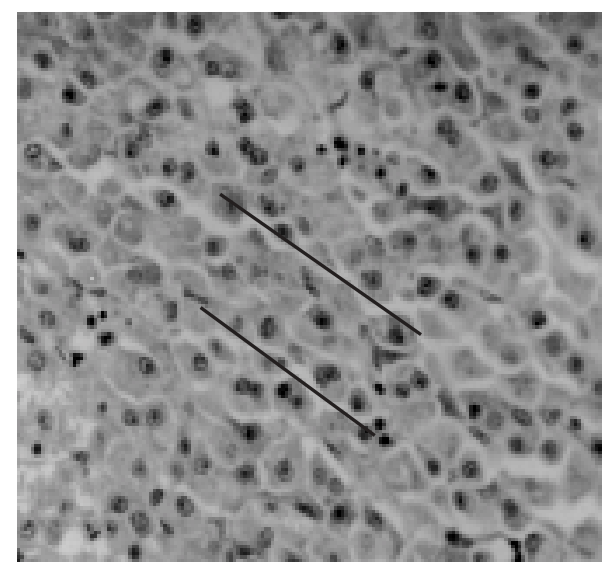

Nos tratamentos com 1,0, 1,5 e 2,0 mL/L ocorreu desarranjo cordonal dos hepatócitos, seus núcleos apresentaram cromatina condensada e diminuição de tamanho, além de fusão celular em algumas regiões e congestão nos sinusóides após $96 \mathrm{~h}$ de exposição (Figura 2). As alterações que ocorreram no fígado do trairão assemelharam-se às descritas por DUTTA et al. (1993) para Heteropneustes fosilis exposto ao malation por 96 horas, por RODRIGUES e FANTA (1998) para Brachydario rerio exposto por oito horas ao dimetoato e por FANTA et al. (2003) para o Corydoras paleatus exposto ao paration metílico. Segundo tais autores, esses inseticidas organofosforados provocaram mudança na granulação citoplasmática dos hepatócitos, reduzindo a eficiência do fígado e levando ao mau funcionamento de outros órgãos do peixe que podem causar a morte. Essas alterações podem indicar a degeneração inicial dos processos intracelulares dos hepatócitos, pois o fígado realiza importantes funções no processo de desintoxicação do animal (DUTTA et al. 1993; HASCHEK e ROUSSEAUX, 1996).

FIGURA 2 - FOTOMICROGRAFIA DO FÍGADO DE TRAIRÃO (Hoplias lacerdae) EXPOSTO A 1,5 mL/L DE FORMALDEÍDO EVIDENCIANDO O DESARRANJO DA ORGANIZAÇÃO CORDONAL DOS HEPATÓCITOS (TRAÇO) AO LONGO DO CAPILAR SINUSÓIDE (S) QUE PARTE DE UMA VEIA (V) - H/E 400X

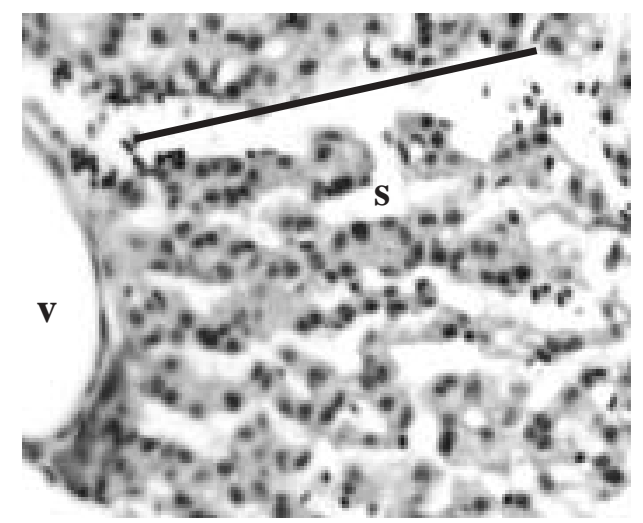


A desorganização do arranjo cordonal também foi observada por DUTTA et al. (1993) em Heteropneus fossilis exposto ao malation e por RIBELLES et al. (1995) em Sparus auratus, exposto ao detergente dodecil sulfato de sódio.

Para JOHNSON et al. (1993), as alterações na histologia dos órgãos aparecem em tempo médio como resposta ao estresse subletal. Assim, como não se conhece o mecanismo de desintoxicação do formaldeído nos peixes é necessário testar outros tempos de exposição e concentrações para o trairão para avaliar a ocorrência de alterações irreversíveis no fígado (MONTENEGRO e FRANCO, 1999).

\title{
4 CONCLUSÃO
}

Pode-se concluir que o formaldeído é moderadamente tóxico para larvas de trairão ( $H$. lacerdae), podendo ser utilizado para terapêutica ou profilaxia de parasitos nas concentrações de 0,5; 1,0 e $1,5 \mathrm{~mL} / \mathrm{L}$ em banho de curta duração. A exposição a concentrações subletais de formaldeído a 10\%/ L de água provocou pequenas alterações no fígado nas larvas de trairão, porém é necessário cuidado com a utilização do formaldeído nos tratamentos de parasitos devido sua toxicidade aguda.

\begin{abstract}
ACUTE TOXICITY AND LIVER HISTOPATHOLOGY IN LARVAE OF THE FRESHWATER FISH (Hoplias lacerdae) EXPOSED TO AN AQUEOUS 10\% FORMALDEHYDE SOLUTION

The objective of the present work was to estimate the $50 \%$ lethal concentration (LC (I) ${ }_{50-96 h}$ ) of an aqueous $10 \%$ formaldehyde solution in larvae of the freshwater teleost (Hoplias lacerdae), and to evaluate the histopathological aspects of the liver of larvae exposed to sub acute concentrations. Six increasing formaldehyde concentrations were tested $(0.0,0.5,1.0,1.5,2.0$ and $2.5 \mathrm{~mL} / \mathrm{L})$. After $96 \mathrm{~h}$ of exposure, the surviving larvae from each treatment were collected. The larvae were fixed in Bouin's solution and embedded in plastic paraffin for the obtention of 5- $\mu \mathrm{m}$ thick histological sections and stained with hematoxylin-eosin. The LC (I) ${ }_{50-96 \mathrm{~h}}$ for the larvae was $2.021 \mathrm{~mL} / \mathrm{L}$ and the mortality was $5 \%, 10 \%, 10 \%$ and $20 \%$ for the treatments with $0.5,1.0,1.5$ and $2.0 \mathrm{~mL} / \mathrm{L}$, respectively, after $96 \mathrm{~h}$ of exposure, and $100 \%$ for the $2.5 \mathrm{~mL} / \mathrm{L}$ concentration after $24 \mathrm{~h}$. Treatments with 1.0, 1.5 and $2.0 \mathrm{~mL} / \mathrm{L}$ resulted in the derangement of the cord-like structure of hepatocytes, cell fusion and obstruction of the sinusoids. Aqueous $10 \%$ formaldehyde solution might be used for therapy or prophylaxis in Hoplias lacerdae larvae at concentrations of $0.5,1.0$ and $1.5 \mathrm{~mL} / \mathrm{L}$ due to the low mortality of the fish, but care is necessary because of its moderately acute toxicity.
\end{abstract}

KEY-WORDS: Hoplias lacerdae; TELEOST; ACUTE TOXICITY; LIVER; FORMALDEHYDE.

\section{REFERÊNCIAS}

1 ALTINOK, I. Toxicity and therapeutic effects of chloramine-T for treating Flavobacterium columnare infection of goldfish. Aquaculture, Amsterdan, v. 239, p. 47-56, 2004.

2 ANGUIANO, O.L.; MONTAGNA, C.M.; CHIFFLET DE LIAMAS, M.; GAUNA, L.; PECHEN D‘ANGELO, A.M. Comparative toxicity of parathion in early embryos and larvae of the toad, Bufo arenarum Hensel. Bull. Environ. Contam. Toxicol., New York, v. 52, p. 649-655, 1994.

3 APHA. American Public Health Association. Standard methods for the examination of water and wastewater. $17^{\text {th }}$ ed. Washington, 1991. p. 81-143.

4 BASER, S.; ERKOÇ, F; SELVI, M.; KOÇAK, O. Investigation of acute toxicity of permethrin on guppies Poecilia reticulata. Chemosphere, v. 51, p. 469-474, 2003.

5 BEHMER, O.A.; TOLOSA, E.M.C.; FREITAS NETO, A.G. Manual de técnicas para histologia normal e patológica. São Paulo: EDART, 1976. 241 p.

6 BURRIDGE, L.E.; HAYA, K.; ZITKO, V.; WADDY, S. The lethality of salmosan (Azamethiphos) to american lobster (Homarus americanus) larvea, poslarvae and adults. Ecotox. Environ. Safety, San Diego, v. 43, p. 165-169, 1999. 
7 CETESB. Companhia de Saneamento Ambiental. Métodos de avaliação da toxicidade de poluentes a organismos aquáticos. São Paulo, 1999, p. 149.

8 CRUZ, C.; MACHADO-NETO, J.G.; MENEZES, M.L. Toxicidade aguda do inseticida paration metílico e do biopesticida azadiractina de folhas de neem (Azadirachta indica) para alevino e juvenil de pacu (Piaractus mesopotamicus). Pesticidas: r. ecotoxicol. e meio ambiente, Curitiba, v. 14, p. 93-102, 2004.

9 CRUZ, E.R.; PITOGO, C.L. Tolerance level and histopathological response of milkfish (Chanos chanos) fingerlings to formalin. Aquaculture, Amsterdan, v. 78, n. 2, p. 135-145, 1989.

10 DUTTA, H.M.; ADHIKARI, S.; SINGH, N.K.; ROY, P.K.; MUNSHI, J. S.D. Histopathological changes induced by Malathion in the liver of freshwater catfish Heteropneustes fossilis (Bloch). Bull. Environ. Contam. Toxicol., New York, v. 51, p. 895-900, 1993.

11 EIRAS, J.C. Elementos de ictioparasitologia. Porto: Ed. Fundação Eng. Antônio de Almeida, 1994, 339 p.

12 FAJER-AVILA, E.J.; PARRA, I.A.; AGUILAR-ZARET, G.; CONTRERAS-ARCE, R.; ZALDIVAR-RAMIREZ, J.; BETANCOURT-LOZANO, M. Toxicity of formalin to bullseye puffer fish (Sphoeroids annulatus Jenyns, 1843) and effectiveness to control ectoparasites. Aquaculture, Amsterdan, v. 223, p. 41-50, 2003.

13 FANTA, E.; RIOS, F.S.; ROMÃO, S.; VIANNA, A.C.C.; FREIBERGER, S. Histopathology of the fish Corydoras paleatus contaminated with sublethal levels of organophosphorus in water and food. Ecotox. Environm. Safety, San Diego, v. 54, p. 119-130, 2003.

14 FDA. Food and Drug Administration. Requirements for investigational new animal drugs. Eastern fish health group and the American fisheries society fish health. The FDA Workshop, Auburn, Alabama, 1992. Disponível em:<www.fda.org> Acesso em: 16 Ago 2005.

15 FDA. Food and Drugs Administration. Certain other from new animal drugs: formalin solution. Code of federal regulations. 21CFR, Part, 529.130. Washington, 1998. Disponível em:<www.fda.org > Acesso em: 29 Abr. 2004.

16 FUJIMOTO, R.Y.; CARNEIRO, D. J. Adição de ascorbil polifosfato como fonte de vitamina C em dietas para alevinos de pintado, Pseudoplatystoma coruscans (Agassiz, 1829). Acta Scientiarum, Maringá, v. 23, n. 4, p. $855-861,2001$.

17 HAMILTON, M.A.; RUSSO, R.C.; THURSTON, V. Trimmed Spearman-Karber method for estimating medial lethal concentrations in toxicity bioassays. Environ. Scien. Techonol., lowa, v. 7, p. 714-719, 1977.

18 HASCHEK, W.M.; ROUSSEAUX, C.G. Handbook of toxicology pathology. London: Academic Press, 1996. 127-151 p.

19 HENARES, M.N.P.; MACHADO-NETO, J.G.; CRUZ, C. Toxicidade aguda do sulfato de cobre pentaidratado para o plati (Xiphophorus maculatus). In: JORNADA CIENTÍFICA DE TOXICOLOGIA, CEATOX, 13. Botucatu: 2003,. Anais... Botucatu: Instituto de Biociências/UNESP, 2003. 47 p.

20 IBAMA. Instituto Brasileiro do Meio Ambiente e dos Recursos Naturais Renováveis. Avaliação da toxicidade aguda para peixes. In: MANUAL de testes para avaliação de ecotoxicidade de agentes químicos. Brasília, DF, 1987. parte 3D.

21 JOHNSON, L.L.; STEHR, C.M.; OLSON, O.P.; MYERS, M.S.; PIERCE, S.M.; WIGREN, C.A.; McCAIN, B.B.; VARANASI, U. Chemical contaminants and hepatic lesions in winter flounder (Pleuronectes americanus) from the northeast coast of the United States. Environ. Scienc. Technol., Iowa, v. 27, p. 2759-2771, 1993.

22 JUNG, S.H.; KIM, J.W.; JEON, I.G.; LEE, Y.H. Formaldehyde residues in formalin-treated olive flouder (Paralichys olivaceus), black rockfish (Sebastes schlegeli), and seawater. Aquaculture, Amsterdan, v. 194, p. 253262, 2001.

23 KINKELIN, P.; MICHEL, C.; GHITTINO, P. Tratado de las enfermidades de los peces. Acribia: Zaragoza, 1992. $353 \mathrm{p}$.

24 MARTINS, M.L. Cuidados básicos e alternativas no tratamento de enfermidades de peixes na aqüicultura brasileira. In: RANZANI-PAIVA, M.J.; TAKEMOTO, R.M.; LIZAMA, M.A.P. Sanidade de organismos aquáticos. São Paulo: Editora Varela, 2004, p. 357-70.

25 MAcNIVEN, A.M.; LITTLE, D.C. Development and evaluation of a stress challenge testing methodology for assessment of nile tilapia (Oreochromis niloticus, Linn.) fry quality. Aquacult. Resear., Oxford, v. 32, p. 671679, 2001.

26 MONTENEGRO, R.M.; FRANCO, M. Patologia: processos gerais. 4. ed. São Paulo: Editora. Atheneu, 1999. 320 p. 
27 MURTY, A.S.; RAMANI, A.V.; CHRISTOPHER, K.; RAJABHUSHANAM, B.R. Toxicity of methyl parathion and fensulfathion to the fish Mystus cavasius. Environ. Pollut. (series A), Massachusetts, v. 34, p. 37-46, 1984.

28 NOGA, E.J. Fish disease: diagnosis and treatment. Mosby: North Carolina State University, St. Louis: College of Veterinary Medicine, 1996. 367 p.

29 PAIVA, M.P. Crescimento, alimentação e reprodução de traíra, Hoplias malabaricus (Bloch), no nordeste brasileiro. Fortaleza: Imprensa Universitária da UFC, 1974. 32 p.

30 RACH, J.J.; HOWE, G.E.; SCHREIER, T.M. Safety of formalin treatments on warm and coolwater fish eggs. Aquaculture, Amsterdam, v. 149, p. 183-191. 1997.

31 REARDON, I.S.; HARRELL, R.M. Acute toxicity of formalin and copper sulfate to striped bass firgerlings held in varying salinities. Aquaculture, Amsterdan, v. 87, p. 255-270, 1990.

32 RIBELLES, A.; CARRASCO, M.C.; ROSETY, M.; ALDANA, M. Morphology and histochemical changes in the liver and pancreas of gilthead, Sparus auratus L., induced by acute action of the anionic detergent, sodium dodecyl sulphate. Histol. Histopathol., Murcia, v. 10, p. 781-787, 1995.

33 RODRIGUES, E.L.; FANTA, E. Liver histopathology of the fish Brachydario rerio after acute exposure to sublethal levels of the organophosphate Dimetoato 500. Rev. Bras. Zool., Curitiba, v. 15, p. 441-450, 1998.

34 ROBERTS, R.J. Patologia de los peces. Madri: Ediciones Mundi-Prensa, 1981. p. 367.

35 ROEX, E.W.M.; VAN LANGEN, M.C.T.; VAN GESTEL, C.A.M. Acute toxicity of two compounds with different modes of action to the zebrafish, Danio rerio. Bull. Environ. Contam. Toxicol., New York. v. 68, p. 269-274, 2002.

36 SHAO-NAN, L.; DE-FANG, F. Correlation between biochemical parameters and susceptibility of freshwater fish to malathion. J. Toxicol. Environ. Heath, Washington, v. 48, p. 413-418, 1996.

37 SOUZA, V.L.; LUNARDI, L.O.; VASQUES, L.H.; CASALETTI, L.; NAKAGHI, L.S.O.; URBINATI, E.C. Morphometric alterations in hepatocytes and ultrastructural distribution of liver glycogen in pacu (Piaractus mesopotamicus, Holmberg, 1887) during food restriction an refeeding. Braz. J. Morphol. Sci., São Paulo, v. 18, n. 1, p. 15-20, 2001.

38 SRIVASTAVA, S.J.; SINGH, N.D.; SRIVASTAVA, A.K.; SINHA, R. Acute toxicity of malachite green and its effects on certain blood parameters of catfish, Heteropneustes fossilis. Aquat. Toxicol., Amsterdam, v. 31, p. 241-247, 1995.

39 SRIVASTAVA, S.; SINHA, R.; ROY, D. Toxicological effects of malachite green. Aquat. Toxicol., Amsterdam, v. 66, p. $319-329,2004$.

40 STRAUS, D.L. Comparison of the acute toxicity of potassium permanganate to hybrid striped bass in well water and diluted well water. J. World Aquacult. Soc., Baton Rouge, v. 35, n. 1, p. 55-60, 2004.

41 TORANZO, A.E.; BARJA, J.L.; DOPAZO, C.P.; ROMALDE, J.L. Enfermedades bacterianas y víricas de peces marinos. In: RANZANI-PAIVA, M.J., TAKEMOTO, R.M.; LIZAMA, M.A.P. Sanidade de organismos aquáticos. São Paulo: Editora Varela, 2004, p. 03-52.

42 ZUCKER, E. Standard evaluation procedure: acute toxicity test for freshwater fish., Washington, 1985. 17 p. USEPA publication 540/9-85-006. Disponível em:<www.epa.gov>. Acesso em: 18 mar. 2005. 